\title{
THE MASS DISTRIBUTION OF THE MILKY WAY DEDUCED FROM GLOBULAR CLUSTER DYNAMICS
}

\author{
B. DAUPHOLE AND J. COLIN
}

Observatoire de Bordeaux, URA 352, CNRS/INSU, B.P. 89, F-33270 Floirac, France

\author{
AND \\ M. GEFFERT, M. ODENKIRCHEN AND H.-J. TUCHOLKE \\ Sternwarte der Universität Bonn, Auf dem Hügel 71, D-53121 \\ Bonn, Federal Republic of Germany
}

\begin{abstract}
We present here a new analytical Galactic potential. We used the constraint of galactic globular cluster dynamics compared to their spatial distribution. This was done with the help of the globular clusters' proper motions. The result for the clusters dynamics show a better agreement between orbital parameters and statistical distribution of the studied globular clusters than in previous published potentials. The globular cluster dynamics constrain the mass distribution on a large scale, until $40 \mathrm{kpc}$ from the centre. In this model, the total mass for the Milky Way is $7.910^{11} \mathrm{M}_{\odot}$.
\end{abstract}

\section{Introduction}

The mass distribution of our Galaxy and especially of the dark matter can be revealed by the dynamics of halo objects. Two categories of interesting tracers are distinguished : Globular clusters and Population II stars. Globular clusters present an advantage over halo stars: They are probably nearly all known and are distributed in the whole Galaxy, whereas catalogs of stars are often not homogeneous and biased. Moreover, globular clusters are objects widely observed due to their discernibility in the Galaxy (Webbink, 1988).

Since the pioneering work of Hartwick \& Sargent (1978) on the globular cluster dynamics, many studies have been made with the help of the glob- 


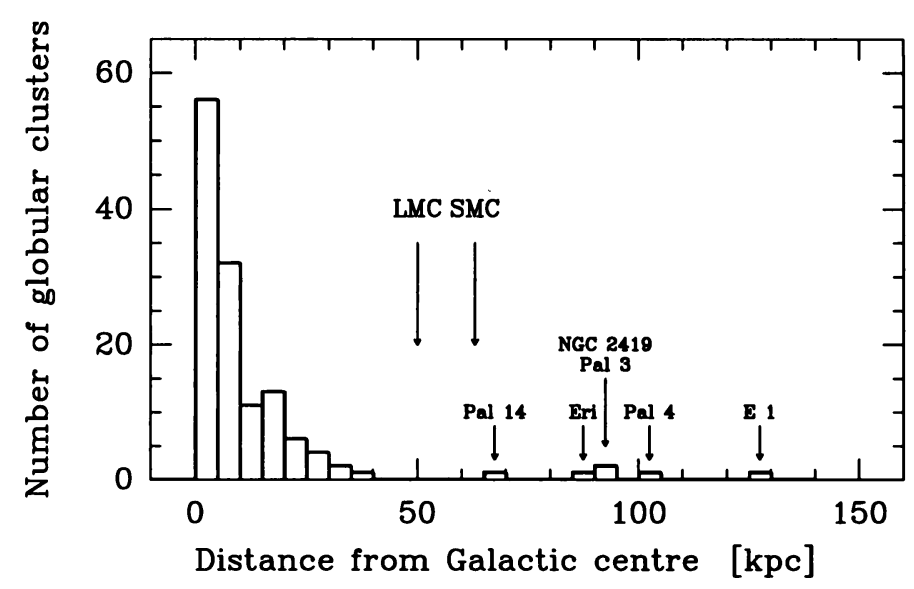

Figure 1. Distribution of observed galactocentric positions for the known globular clusters of the Galaxy. Distances are derived from Peterson (1993).

ular cluster radial velocities. These studies have shown that the mass of our Galaxy ranges between $310^{11} \mathrm{M}_{\odot}$ up to $1310^{11} \mathrm{M}_{\odot}$ (e.g. Miyamoto et al. 1980; Frenk \& White 1980; Innanen et al. 1983; Peterson 1985; Suntzeff et al. 1985; Olszewski et al. 1986; Little \& Tremaine 1987; Thomas 1989; Zaritsky et al. 1989; Kulessa \& Lynden-Bell 1992). But until now, these studies used the globular cluster radial velocities and distances alone.

Absolute proper motions for 26 galactic globular clusters are now known (Dauphole et al., 1994). For 12 of them, proper motions were calibrated to extragalactic objects. It is therefore possible to investigate their dynamical behavior in a realistic available potential. We show here that, to be consistent with the observation of the spatial distribution, one has to constrain the mass distribution of the Milky Way at a large distance from the centre.

\section{The Space Velocities of Globular Clusters : a New Constraint to the Galactic Potential}

From the distances, radial velocities and proper motions of the clusters, we obtained their spatial velocities. Previous work which took into account the proper motions have shown that some of them have large apocentres, sometimes greater than $40 \mathrm{kpc}$ (Allen \& Martos 1988; Allen 1990; Brosche et al. 1991; Odenkirchen \& Brosche 1992; Allen \& Santillan 1993).

However, during their orbital motion these objects spend a large part of their orbital period at distances near their apocentre. An example of orbital motion and time spent by NGC 4147 is given in Odenkirchen \& Brosche (1992) : This cluster is at distance greater than $40 \mathrm{kpc}$ during $\approx 70 \%$ of its 
orbital period. So, statistically, we will observe the majority of clusters at a galactocentric distance near their apocentric distance.

Remarkably, the distance from the Galactic centre of the clusters in the Milky Way presents a distribution (Fig. 1) with two groups : The first, containing 129 clusters, shows a decrease then a gap after $40 \mathrm{kpc}$, and the second one, with only 6 clusters, located beyond the Magellanic Clouds, is sparse and has probably a different nature. Since the probability to observe clusters is larger at distances near their apocentre, the distribution of the Fig. 1 indicates that the globular clusters may not have apocentres larger than the observed limit of $40 \mathrm{kpc}$. Otherwise we should observe some beyond the end of the observed distribution.

This fact imposes a new constraint to the potential of our Galaxy at large distance, i.e., clusters with known spatial velocities should not have apocentric distances greater than $40 \mathrm{kpc}$.

\section{The Potential Model}

Previous models for the potential of our Galaxy give unrealistic orbits for the clusters, in view of their observed spatial distribution. We have built a realistic Galactic potential which reconciles the cluster motion and the statistical distribution (Dauphole \& Colin, 1994). The total potential is composed of a bulge, a disc and a halo :

$$
\Phi(\varpi, z)=\Phi_{\mathrm{b}}(r)+\Phi_{\mathrm{d}}(\varpi, z)+\Phi_{\mathrm{h}}(r)
$$

The bulge and the halo are represented by two Plummer's spheres, and the disc by a Kuzmin's disc. The adopted analytical forms are from Miyamoto \& Nagai (1975):

$$
\begin{gathered}
\Phi_{\mathrm{b}, \mathrm{h}}(r)=-\frac{G M_{\mathrm{b}, \mathrm{h}}}{\left[r^{2}+b_{\mathrm{b}, \mathrm{h}}^{2}\right]^{1 / 2}} \\
\Phi_{\mathrm{d}}(\varpi, z)=-\frac{G M_{\mathrm{d}}}{\left[\varpi^{2}+\left[a_{\mathrm{d}}+\left(z^{2}+b_{\mathrm{d}}^{2}\right)^{1 / 2}\right]^{2}\right]^{1 / 2}}
\end{gathered}
$$

The adjusted constants $\left(M_{\mathrm{b}}, M_{\mathrm{d}}, M_{\mathrm{h}}, b_{\mathrm{b}}, b_{\mathrm{d}}, b_{\mathrm{h}}\right.$ and $\left.a_{\mathrm{d}}\right)$ and derived parameters are presented in Tab. 1. In this model, the resulting Galactic mass is only $7.910^{11} \mathrm{M}_{\odot}$. The combined mass of the disc and the bulge is $9.310^{10} \mathrm{M}_{\odot}$. In Fig. 2 it is shown that the rotation curve derived from this model is in good agreement with the data of the work from Brand \& Blitz (1993). 


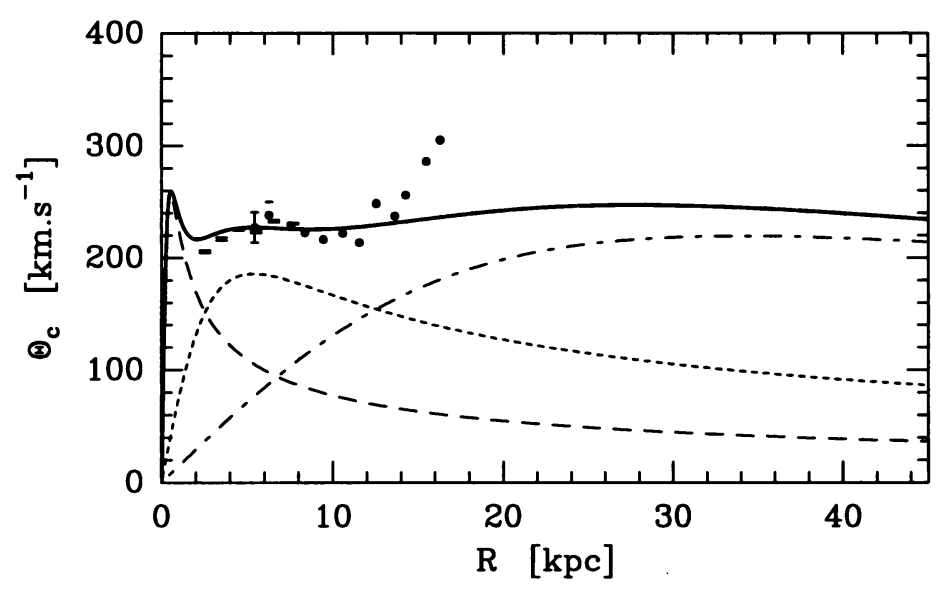

Figure 2. Rotation curve of the model (solid line) with its contributions from the bulge (dashed line), the disc (dotted line) and the halo (dashed-dotted line). Observational data with error bars are from Brand \& Blitz (1993).

We computed orbital parameters for the most critical globular clusters, that is clusters with large space motion, with an integration scheme of Bulirsch-Stoer, and compared them to the ones computed in the model of Allen \& Martos (1988) (hereafter AM88). Results are given in Tab. 2 along with the uncertainties on the apocentre. For each cluster we present its observed distance from the centre (column 2) and the apocentric distance reach in the AM88 model (column 3) and in our model (column 4). The errors on apocentres of the fourth column were computed as follows : We added and subtracted the measurement errors on proper motion, distance and radial velocities, then we computed the orbit and we kept the greater and the lower value obtained.

Apocentres computed in our model are smaller than in the AM88 model. However, two of them still have apocentre greater than the imposed limit, but less pronounced than in the model of Allen \& Martos. Errors on the measured data can explain this disagreement. However, the global trend of this model is to reduce the apocentric distances for the clusters which have large apocentre. For clusters with smaller apocentric distances, the gain compared to other models is not really large, but our model also decreases the apocenters of these clusters. The advantage of our model is that the probability to observe them at their current position (column 2 of Tab. 2) is increased. 
TABLE 1. Adjusted constants and computed local parameters.

\begin{tabular}{ll}
\hline Distance Sun-Galactic centre (adopted) & $\mathrm{R}_{\odot}=8.0 \mathrm{kpc}$ \\
Local circular velocity (adopted) & $\mathrm{V}_{\mathrm{lsr}}=225 \mathrm{~km} \cdot \mathrm{s}^{-1}$ \\
Bulge constants & $\mathrm{M}_{\mathrm{b}}=1.395510^{10} \mathrm{M}_{\odot}$ \\
& $\mathrm{b}_{\mathrm{b}}=0.35 \mathrm{kpc}$ \\
Disc constants & $\mathrm{M}_{\mathrm{d}}=7.908010^{10} \mathrm{M}_{\odot}$ \\
& $\mathrm{a}_{\mathrm{d}}=3.55 \mathrm{kpc}$ \\
& $\mathrm{b}_{\mathrm{d}}=0.25 \mathrm{kpc}$ \\
Halo constants & $\mathrm{M}_{\mathrm{h}}=6.977610^{11} \mathrm{M}_{\odot}$ \\
& $\mathrm{b}_{\mathrm{h}}=24.0 \mathrm{kpc}$ \\
Total mass & $\mathrm{M}_{\mathrm{T}}=7.90810^{11} \mathrm{M}_{\odot}$ \\
Local density & $\rho_{0}=0.143 \mathrm{M}_{\odot} \cdot \mathrm{pc}^{-3}$ \\
Rotation constants & $\mathrm{A}=14.25 \mathrm{~km} \cdot \mathrm{s}^{-1} \cdot \mathrm{kpc}^{-1}$ \\
& $\mathrm{~B}=-13.89 \mathrm{~km} \cdot \mathrm{s}^{-1} \cdot \mathrm{kpc}^{-1}$ \\
Local escape velocity & $v_{\mathrm{e}}=573.5 \mathrm{~km} \cdot \mathrm{s}^{-1}$ \\
\hline
\end{tabular}

TABLE 2. Observed positions and apocentres reached in Allen \& Martos (1988) potential and in our model. Positions are derived from Peterson's (1993) data.

\begin{tabular}{|c|c|c|c|}
\hline \multirow[t]{2}{*}{ Name } & \multirow[t]{2}{*}{$\begin{array}{l}R_{o b s} \\
(k p c)\end{array}$} & \multicolumn{2}{|c|}{$\begin{array}{l}R_{\text {apo }} \\
(\mathrm{kpc})\end{array}$} \\
\hline & & AM88 & This model \\
\hline NGC 4147 & 20.9 & 84.7 & $52.0 \pm \underset{24.3}{\infty}$ \\
\hline NGC 5466 & 16.2 & 90.1 & $51.9 \pm 346.9$ \\
\hline NGC 5904 & 6.2 & 69.8 & $37.3 \pm \underset{10.0}{17.1}$ \\
\hline NGC 6779 & 9.4 & 53.5 & $33.4 \pm \begin{array}{c}148.6 \\
15.8\end{array}$ \\
\hline NGC 6934 & 12.1 & 53.3 & $31.7 \pm{ }_{12.5}^{28.0}$ \\
\hline NGC 7078 & 10.5 & 66.6 & $37.0 \pm \begin{array}{l}95.4 \\
19.0\end{array}$ \\
\hline NGC 7089 & 10.6 & 42.0 & $27.7 \pm \quad \begin{array}{l}30.8 \\
11.2\end{array}$ \\
\hline
\end{tabular}

\section{Conclusion}

We have constructed a mass distribution model for the Milky Way in accordance with the classical observational constraints (rotation curve, local perpendicular force, local density and Oort's constants), and with the dynamics of the galactic globular clusters. This was possible due to the knowledge of the proper motions for 26 clusters. The new constraint introduced 
here is a large scale constraint, until $40 \mathrm{kpc}$, since there is a deficiency of clusters beyond this limit. It can be explained if the clusters do not have apocentric distances greater than $40 \mathrm{kpc}$, otherwise we should observe some of them beyond this limit. The derived model is completely analytical and is well suited for orbit computation. Finally to constrain the dynamics of galactic globular clusters to their observed distribution, a total mass for the Galaxy of only $7.910^{11} \mathrm{M}_{\odot}$ seems to be sufficient.

\section{Acknowledgements}

We are grateful to Ch. Ducourant who checked this text, and would like to thank colleagues for their help in the typesetting.

\section{References}

Allen, C. (1990), Rev.Mex. Astron. Astrophis., 20, pp. 67-72

Allen, C., Martos, M.A. (1988), Rev.Mex. Astron. Astrophis., 16, pp. 25-36

Allen, C., Santillan, A. (1993), Rev.Mex. Astron. Astrophis., 25, pp. 39-50

Brand, J., Blitz, L. (1993), $A \& A$, 275, pp. 67-90

Brosche, P., Tucholke, H.-J., Klemola, A.R., Ninković, S., Geffert, M., Doerenkamp, P. (1991), $A J$, 102, pp. 2022-2027

Dauphole, B., Colin, J. (1994), $A \cup A$, submitted.

Dauphole, B., Geffert, M., Colin, J., Ducourant, Ch., Odenkirchen, M. (1994), $A \cup A S$, submitted.

Frenk, C.S., White, S.D.M. (1980), MNRAS, 193, pp. 295-311

Hartwick, F.D.A., Sargent, W.L.W. (1978), $A p J, 221$, pp. 512-520

Innanen, K.A., Harris, W.E., Webbink, R.F. (1983), $A J$, 88, pp. 338-360

Kulessa, A.S., Lynden-Bell, D. (1992), MNRAS, 255, pp. 105-118

Little, B., Tremaine, S. (1987), $A p J$, 320, pp. 493-501

Miyamoto, M., Nagai, R. (1975), PASJ, 27, pp. 533-543

Miyamoto, M., Satoh, C., Ohashi, M. (1980), $A \& A$, 90, pp. 215-223

Odenkirchen, M., Brosche, P. (1992), Astron. Nachr., 313, pp. 69-81

Olszewski, E.W., Peterson, R.C., Aaronson, M. (1986), ApJ, 302, pp. L45-L48

Peterson, R.C. (1985), ApJ, 297, pp. 309-313

Peterson, R.C. (1993), ESO preprint 932, pp. 13-18

Suntzeff, N., Olszewski, E., Stetson, P.B. (1985), AJ, 90, pp. 1481-1485

Thomas, P. (1989), MNRAS, 238, pp. 1319-1343

Webbink, R.F. (1988), iñ IAU Symposium 126, The Harlow-Shapley Symposium on Cluster Systems in Galaxies, J.E. Grindlay and A.G. Davis Philip, eds., Reidel, Dordrecht, pp. $49-60$

Zaritsky, D., Olszewski, E.W., Schommer, R.A., Peterson, R.C., Aaronson, M. (1989), $A p J, 345$, pp. 759-769 